\title{
La influencia del entorno próximo sobre la persistencia en la práctica de actividad físico-deportiva
}

\section{Influence of environment on persistence in the sport physical activity}

\section{A influencia do entorno sobre a persisitencia na pratica da atividade físico- esportiva}

\author{
Pedro Antonio Sánchez Miguel, Francisco Miguel Leo Marcos, David Sánchez Oliva, \\ Diana Amado Alonso y Tomás García Calvo
}

Universidad de Extremadura.

\begin{abstract}
Resumen: El objetivo del presente estudio es conocer la importancia que adquieren los otros significativos, padres, entrenador y compañeros, sobre la persistencia o cese de la práctica deportiva en jóvenes estudiantes. La muestra está formada por 958 jóvenes participantes de deportes colectivos, con edades comprendidas entre los 11 y 16 ańos, pertenecientes a la Comunidad Autónoma de Extremadura. Se emplearon instrumentos para valorar la implicación de los padres en la práctica deportiva, así como el clima motivacional de compañeros y entrenador. Los resultados nos muestran una capacidad predictiva de la implicación al club de los padres y el apoyo sobre la continuidad en el deporte, así como el clima de la tarea por el entrenador. Finalmente se discuten los resultados y se muestran limitaciones y prospectivas de futuro.

Palabras claves: Motivación, otros significativos, deportes colectivos.

Abstract: The aim of this study is to know the importance of other significatives, parents, coaches and peers, on persistence or dropped out of the sporting practice in young students. The sample was formed by 958 young participants of team sports, ranging in age from 11 to 16 years old, belonged to the Region of Extremadura. We used different instruments to assess parents'
\end{abstract}

involvement in the sport practice, as well as peer and coaches motivational climate. Results showed a predictive capacity of parents' club involvement and support on persistence in sport, as well as coaches' mastery climate. Finally, results are discussed and show limitations and future prospective. Keywords: Motivation, other significatives, team sports.

Resumo: O objetivo do presente estudo é de conhecer a importância que adquirem os outros significativos, país, treinadores e companheiros sobre a persistência o fim da pratica esportiva em jovens estudantes. A mostra esta formada por 958 jovens participantes de esportes coletivos, com idades compreendidas entre 11 e 16 anos, pertencentes da Comunidad Autónoma de Extremadura. Foram aplicados instrumentos para avaliar a implicação dos pais na prática esportiva, assim como no clima motivacional dos companheiros e treinadores. Os resultados mostraram uma capacidade preditiva da implicação do clube e dos pais e sobre a continuidade no esporte, assim como o clima da tarefa do treinador. Finalmente se discute os resultados e se mostram as limitaçóes e perspectivas de futuro.

Palavras chave: Motivaçáo, outros significados e esportes coletivos.

\section{Introducción}

La promoción de actividad física en etapas tempranas es un objetivo a alcanzar por parte de la comunidad educativa y política de todos los países (Telama y cols., 2005), teniendo en cuenta que los niveles de actividad física y deportiva en edad escolar están relacionados con su posterior práctica en la etapa adulta (Tammelin, Näyhä, Hillas y Järvelin, 2003; Vanreusel y cols., 1997). Recientemente, se están desarrollando líneas de investigación dónde se demuestra que el entorno próximo, como son los padres, entrenadores, profesores, compańeros de juego... del joven deportista, llevan a cabo un papel fundamental en la adquisición de hábitos saludables y comportamientos adaptativos (Ferrer-Caja y Weiss, 2002; Jowett y Timson-Katchis, 2005; Weiss y Fretwell, 2005).

Dr. Pedro Antonio Sánchez Miguel

Facultad de Ciencias del Deporte, Universidad de Extremadura.

Avenida de la Universidad, S/N, 10071 Cáceres. E-mail: pesanchezm@unex.es
En primer lugar, hay que destacar a la familia, que es el núcleo donde se producen las primeras experiencias de socialización deportiva (Duda, Ntoumanis, Mahoney, Larson y Eccles, 2005), por ello las actitudes que mantenga este significativo frente a la sociedad y principalmente frente al deporte serán determinantes. Diversos estudios demuestran una estrecha relación entre las percepciones de los jóvenes sobre el interés de los padres hacia el deporte y los niveles de participación deportiva de los hijos (Brustad, 1996; Jowett y Timson-Katchis, 2005).

Pero no sólo los padres son influyentes en la adopción de comportamientos del joven deportista, sino que también el entrenador es un elemento muy condicionante, o más bien, su manera de focalizar la actividad, que determinará una mayor o menor predisposición de los niños a la realización de actividades deportivas (Conroy y Coatsworth, 2006; Gallimore y Tharp, 2004; Mageau y Vallerand, 2003). Muchos en- 
trenadores ejercen una influencia positiva sobre la motivación de los deportistas y su bienestar, promoviendo la diversión, satisfacción y el afecto positivo (Boixadós, Cruz, Torregrosa y Valiente, 2004; Sousa, Arevalo, Ramis, Torregrosa, Vilches y Cruz, 2006); además, fomentan una relación positiva entre los compañeros (Ommudsen, Roberts, Lemyre y Miller, 2005) y favorecen la práctica deportiva continua (Balaguer, Duda, Atienza y Mayo, 2002; Smith, Fry, Ethington y Li, 2005), lo que produce una disminución en la intención de dejar el deporte (Balaguer, Duda y Crespo, 1999). Sin embargo, conductas inapropiadas por parte del entrenador como disminuir el apoyo social y el feedback positivo, e incrementar un feedback orientado al castigo, conducen a consecuencias desadaptativas (Balaguer y cols., 2002; Bartholomew, Ntoumanis y Thøgersen-Ntoumani, 2009; Smith y cols., 2005) como puede ser el cese de la práctica deportiva (Cervelló, Escartí y Guzman, 2007; Sarrazin, Vallerand, Guillet, Pelletier y Cury, 2002).

En esta línea, autores como García Calvo, Leo, Martín y Sánchez Miguel (2008) defendían que el comportamiento inadecuado de padres y entrenadores (excesiva presión, comportamiento directivo, falta de reconocimiento al esfuerzo y progreso...), disminuía el compromiso deportivo y podía conducir al abandono de la práctica deportiva. De esta manera, se ha demostrado que las consecuencias negativas en el contexto deportivo aumentan cuando los jóvenes perciben excesiva presión por parte de los entrenadores y otros adultos cercanos a ellos (Fraser-Thomas y Côté, 2009).

Además de la importancia de los padres y entrenadores sobre la adherencia al deporte de los jóvenes, el grupo de compañeros de juego también emergen como importantes socializadores en el desarrollo del joven. Los hallazgos encontrados respecto a ello, indican que la percepción de los individuos de su propia competencia deportiva, está fuertemente relacionada con la idea de ser valorado y aceptado por sus compañeros (Weiss y Duncan, 1992). Recientemente, Moreno Murcia, López de San Román, Martínez, Alonso y González-Cutre (2008) encontraron en una muestra de 394 practicantes de actividad física no competitiva, que la generación de un clima positivo de aprendizaje, esfuerzo y colaboración entre los compañeros, es una herramienta eficaz para favorecer aspectos positivos como la percepción de competencia, autonomía y relaciones sociales, además de fomentar la diversión en un contexto no competitivo. Por otro lado, Ntoumanis, Vazou y Duda (2007) destacan la importancia que presentan los compañeros, ya que pueden predecir consecuencias motivacionales independientemente del entrenador o los padres. Finalmente, si atendemos a la importancia que adquiere la diversión (Weiss y Ferrer-Caja, 2002) y la amistad (Weiss y Smith, 2002) en el compromiso deportivo de los jóvenes y una práctica duradera en el contexto deportivo, se puede entender la importancia de los compañeros de juego en la persistencia en el deporte.

De acuerdo a todo lo expuesto anteriormente, la Teoría de las Metas de Logro (Nicholls, 1989; Ames, 1992), se muestra consistente para examinar las relaciones entre los padres (Papaioannou, Ampatzoglou, Kalogianis y Sagovists, 2008), entrenadores (Ommundsen y Roberts, 1999) y compañeros (Smith, Balaguer y Duda, 2006) con respecto al joven deportista. Esta teoría explica que existen dos tipos de orientación motivacional en función del concepto de habilidad percibida. El primero es la orientación hacia el ego, consistente en valorar la habilidad comparándose con otras personas, dando más importancia al resultado de nuestro comportamiento que al esfuerzo y a la ejecución (Nicholls, 1992). Por el contrario, la orientación a la tarea se caracteriza por valorar la habilidad en función de uno mismo, así como por ensalzar el esfuerzo y la ejecución por encima de los resultados. Asimismo, esta teoría establece que en función de las características motivacionales que perciba el sujeto en las personas de su entorno, se puede diferenciar entre un clima motivacional que implica al ego o a la tarea en ese contexto (Ames, 1992).

Por todo ello, el objetivo del presente estudio es conocer la importancia que tienen los otros significativos, padres, entrenadores y compañeros, sobre la continuidad o abandono de la práctica deportiva. De esta manera, se plantean como hipótesis que un mayor apoyo e implicación por parte de los padres estará asociado con la persistencia en la práctica deportiva. Asimismo, se postula que la continuidad en la práctica deportiva va a estar positivamente relacionada con el clima tarea del entrenador y de los compañeros.

\section{Método}

\section{Participantes}

La muestra de nuestra investigación pertenece a una población de deportistas extremeños con edades comprendidas entre los 11 y los 16 ańos $(M=13.42 ; S D=1.32)$, practicantes de los deportes colectivos de baloncesto $(N=182)$, balonmano $(N=68)$, fútbol $(N=590)$ y voleibol $(N=118)$. El total de la muestra lo componían 958 jóvenes participantes de género masculino $(N=732)$ y femenino $(N=226)$. Todos los participantes realizaban práctica deportiva en los JUDEX (Juegos Deportivos Extremeños), por lo que poseían una ficha federativa para consultar todos sus datos. En relación al número de abandonos, 767 participantes han continuado en la práctica deportiva, que representaba un $80,1 \%$ del total de participantes, mientras que 191 deportistas abandonaron, que se correspondía con el 19,9\% del total. Todos ellos fueron seleccionados mediante un procedimiento de casos por muestreo aleatorio por conglomerados. En la siguiente tabla se muestran los abandonos y no abandonos especificados por deporte. 
Tabla 1. Total de participantes en la investigación

\begin{tabular}{lccc}
\hline & Participantes & Abandonos & No abandonos \\
\hline Baloncesto & 182 & 143 & 39 \\
Balonmano & 68 & 62 & 6 \\
Voleibol & 118 & 91 & 27 \\
Fútbol & 590 & 471 & 119 \\
\hline
\end{tabular}

\section{Instrumentos}

Participación de los padres en la práctica deportiva. Para su medición se ha utilizado la versión traducida al castellano del Parental Involvement Sport Questionaire (PISQ: Lee y Mclean, 1997) empleada en estudios previos por Torregrosa y cols. (2005) y Torregrosa y cols. (2007). Dicho cuestionario cuenta con un total de 20 ítems que se agrupan en cuatro factores principales: comportamiento directivo (5 ítems, i.e.: Tus padres antes de los partidos, te dicen cómo has de jugar), apoyo y comprensión ( 6 ítems, i.e.: Tus padres te elogian por lo que has hecho bien, aunque el partido se haya perdido), implicación activa ( 5 ítems, i.e.: Tus padres tienen un papel activo en el funcionamiento del club) y presión (4 ítems, i.e..: Tus padres te presionan para que entrenes mejor). Los dos primeros factores hacen referencia al clima que implica a la tarea y los dos últimos al clima que implica al ego.Clima motivacional de los entrenadores. Se ha utilizado la versión traducida al castellano del Perceived Motivational Climate in Sport Questionnaire (PMSCQ - 2: Newton, Duda y Yin, 2000). Este cuestionario consta de 21 ítems, agrupados en clima que implica a la tarea (12 ítems, i.e.: El entrenador favorece que los jugadores se ayuden en los entrenamientos y partidos), y clima que implica al ego (9 ítems, i.e.: El entrenador motiva a los jugadores cuando juegan mejor que sus compañeros.

Clima motivacional percibido en los compañeros de juego. Se ha empleado la versión traducida al castellano del Peer Motivational Climate in youth sport Questionaire (PEERMCYSQ: Ntoumanis y Vazou, 2005). Se trata de una versión reducida, donde tan sólo se han utilizado 15 de los 21 ítems propuestos en la versión original.. Este instrumento consta de dos factores principales denominados clima que implica al ego (4 ítems, i.e.: En mi grupo de entrenamiento, los compañeros(as) quieren ser los mejores del grupo y clima que implica a la tarea (11 ítems, i.e.: En mi grupo de entrenamiento, los compañeros(as) animan a los otros compañeros a esforzarse).

Medida del abandono deportivo. El abandono deportivo se valoró mediante el análisis de las licencias federativas que se realizaron la siguiente temporada en la que se llevó a cabo la toma de datos. Así, a partir del mes de octubre se comprobó mediante los archivos de las Federaciones Extremeńas de Fútbol, Baloncesto, Voleibol y Balonmano, quiénes habían sido inscritos y se consideraron como "No Abandono". Por otro lado, aquellos que no aparecieron registrados en la federación, fueron considerados como "Abandono". En este sentido, hay que ser prudentes ya que, puede ser que aparezcan participantes como "abandonos" y éstos hayan dejado la práctica federativa, pero estén participando en otro nivel, o incluso algunos participantes que se hayan trasladado de localidad y estén compitiendo/participando en otra comunidad autónoma. A pesar de esta indicación, se puede considerar que la mayoría de los "abandonos" presentes en esta investigación, han dejado realmente la práctica deportiva, o al menos la competitiva que es la que se desea examinar.

\section{Procedimiento}

Se realizó un protocolo estandarizado con el objetivo de que la recogida de los datos fuera lo más similar posible durante todo el proceso. En primer lugar, se contactó con los entrenadores y monitores de diferentes equipos que podrían formar el conjunto de participantes de la investigación. También se informó a los deportistas de que su participación era voluntaria y las respuestas serían tratadas confidencialmente. Los participantes rellenaron los cuestionarios en el vestuario, sin la presencia del entrenador, de manera individual y en un clima que les permitía concentrarse sin tener ningún tipo de distracción. El investigador principal estuvo presente en el momento en que los sujetos completaban los cuestionarios. La recogida de datos se realizó de marzo a junio, considerando que son estas fechas las más apropiadas para el estudio y análisis de nuestras variables.

Posteriormente, llevamos a cabo el análisis del abandono deportivo de los participantes. A inicios de la temporada siguiente, en el mes de septiembre, se comenzó a analizar las licencias federativas de todos los participantes, que fueron facilitadas por las diferentes federaciones. Una vez comprobadas las diferentes licencias, se registró a los individuos que continuaron en la práctica deportiva y a los que abandonaron. Los participantes que no estaban inscritos en la federación correspondiente, se contactó con el entrenador y con ellos personalmente, para contrastar dicho abandono. Se destaca que de los 191 jóvenes que abandonaron la práctica deportiva federada de los deportes analizados, no todos cesaron en la práctica de algún deporte. De esta manera, 14 jóvenes continuaron en la práctica de otras modalidades deportivas (cinco chicos de fútbol cambiaron a baloncesto, dos chicos de fútbol empezaron a practicar ciclismo, un participante de fútbol optó por tenis, otro del mismo deporte cambió a atletismo, un chico de voleibol cambió a tenis, dos chicos de balonmano y baloncesto cambiaron a fútbol, un chico de baloncesto empezó a practicar piragüismo, y finalmente una chica de baloncesto cambió por kárate).

\section{Análisis de datos}

Se empleó el programa estadístico SPSS 15.0 para el tra- 
tamiento de los datos. Las técnicas estadísticas que se emplearon fueron el análisis descriptivo, análisis de fiabilidad, análisis de varianza y análisis discriminante. Se emplea como variable dependiente el abandono de la práctica deportiva (continuaban la próxima temporada en la práctica deportiva o cesaban en la práctica), mientras que las variables independientes serán los climas motivacionales de los otros significativos (padres, compañeros y entrenadores).

\section{Resultados}

En la Tabla 2, se muestran los resultados correspondientes al análisis descriptivo, curtosis y asimetría de los climas motivacionales de padres, entrenadores y compañeros. En ellos, se puede apreciar cómo los valores medios de aspectos adap- tativos como el apoyo al club de los padres $(M=4.107 ; S D$ $=.807)$, son muy superiores a las puntuaciones que presentan conductas desadaptativas como la presión $(M=2.253 ; S D=$ 1.133) y el comportamiento directivo $(M=3.175 ; S D=1.091)$. Asimismo, se observa que los valores del clima tarea, tanto en los entrenadores $(M=4.301 ; S D=.685)$ como en los compañeros $(M=4.183 ; S D=.744)$, son bastante más elevados que los valores ego de los mismos significativos $(M=2.226$; $S D=$ .994 y $M=3.333$; $S D=1.090$ respectivamente). Por otro lado, la normalidad de los datos se manifiesta adecuada mediante los valores de la curtosis y asimetría.

Además, se aprecia que todas las variables presentan valores aceptables de fiabilidad en los índices alfa de Cronbach (mayores a .70), lo que nos indica una adecuada consistencia interna de los instrumentos de evaluación utilizados.

Tabla 2. Estadísticos descriptivos, curtosis y asimetría de los climas motivacionales de los otros significativos

\begin{tabular}{|c|c|c|c|c|c|}
\hline Variables & $\mathrm{M}$ & SD & $\alpha$ & Curtosis & Asimetría \\
\hline \multicolumn{6}{|l|}{ Padres } \\
\hline Comportamiento Directivo & 3.175 & 1.091 & .859 & -.842 & -.125 \\
\hline Implicación Club & 3.044 & 1.146 & .771 & -.901 & -.036 \\
\hline Apoyo Padres & 4.107 & .807 & .794 & 1.161 & -1.096 \\
\hline Presión padres & 2.253 & 1.133 & .702 & -.375 & .759 \\
\hline \multicolumn{6}{|l|}{ Compañeros } \\
\hline Clima Tarea & 4.183 & .744 & .747 & 1.963 & -1.187 \\
\hline Clima Ego & 3.333 & 1.090 & .797 & -.622 & -.325 \\
\hline \multicolumn{6}{|l|}{ Entrenador } \\
\hline Clima Tarea & 4.301 & .685 & .864 & 3.181 & -1.598 \\
\hline Clima Ego & 2.226 & .994 & .831 & .012 & .809 \\
\hline
\end{tabular}

Con el objetivo de conocer el grado y la dirección de las relaciones entre las percepciones de los climas motivacionales de los entrenadores, compañeros, así como la implicación, apoyo, presión y comportamiento de los padres, realizamos un análisis correlacional (Tabla 3). Así, se puede destacar cómo la presión de los padres está asociada con el clima ego del entrenador y los compañeros, mientras que el apoyo de los padres presenta una relación positiva con la presión, el clima tarea y ego de los compańeros y el clima tarea del entrenador. El clima tarea de los compañeros mantiene una relación positiva con el clima tarea del entrenador, mientras que mantiene una relación negativa con el clima ego de los compañeros y del entrenador. Este aspecto nos indica que los constructos están teóricamente bien relacionados.

Tabla 3. Correlaciones entre los climas motivacionales de padres, entrenadores y compañeros

\begin{tabular}{|c|c|c|c|c|c|c|c|c|}
\hline & 1 & 2 & 3 & 4 & 5 & 6 & 7 & 8 \\
\hline 1. Comportamiento directivo & & & & & & & & \\
\hline 2. Implicación club & $.462(* *)$ & - & & & & & & \\
\hline 3. Apoyo padres & $.441(* *)$ & $.397(* *)$ & - & & & & & \\
\hline 4. Presión padres & $.543(* *)$ & $.373(* *)$ & $.193(* *)$ & - & & & & \\
\hline 5. Clima tarea comp. & $.138\left(^{* *}\right)$ & $.209\left(^{* *}\right)$ & $.328\left(^{* *}\right)$ & -.009 & - & & & \\
\hline 6. Clima ego comp. & $\left..250{ }^{(* *}\right)$ & $.072(*)$ & $.075\left(^{*}\right)$ & $.315(* *)$ & $-.091(* *)$ & - & & \\
\hline 7.Clima tarea entrenador & $.119(* *)$ & $\left..180{ }^{* *}\right)$ & $.348(* *)$ & -0.05 & $.453(* *)$ & -.001 & - & \\
\hline 8.Clima ego entrenador & $.218(* *)$ & $.098(* *)$ & -.016 & $.453(* *)$ & $-.105(* *)$ & $.415(* *)$ & $-.299(* *)$ & - \\
\hline
\end{tabular}

${ }^{* *} p<.01 ;{ }^{*} p<.05$ 
En la Tabla 4, se muestran los resultados obtenidos tras el análisis de varianza de los climas motivacionales de los otros significativos en función del abandono deportivo. En primer lugar, se puede destacar que existen diferencias significativas entre los que abandonaron y los que continuaron en la práctica, en el apoyo de los padres $(p=.039)$ y el clima tarea del entrenador $(p=.024)$. Así, los que persistieron en la práctica

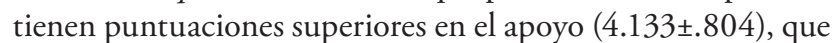
los que abandonaron (3.998 \pm .812$)$. Igualmente, los participantes que continuaron presentan valores superiores en el clima tarea del entrenador (4.326士.649), que aquellos que la temporada siguiente decidieron no continuar (4.201土.806). En esta tabla también se reflejan los resultados encontrados tras la realización del análisis discriminante teniendo como variable dependiente al abandono deportivo, y como variables independientes a los climas de los otros significativos. En ella se destaca cómo la implicación al club de los padres emerge como el principal predictor de la continuidad en la práctica deportiva, seguido por el clima tarea del entrenador y el apoyo de los padres. Sorprendentemente, en último lugar aparece como variable predictora de la continuidad en el deporte la presión por parte de los padres en último lugar.

Tabla 4. Análisis de varianza y análisis discriminante de los climas motivacionales de los otros significativos en función del abandono deportivo.

\begin{tabular}{lcrrrrr}
\hline Variables & $\begin{array}{c}\text { Abandona } \\
(N=191)\end{array}$ & $\begin{array}{c}\text { No Abandona } \\
(N=767)\end{array}$ & \multicolumn{1}{c}{$M C$} & \multicolumn{1}{c}{$F$} & $p$ & Coeficiente Discriminante \\
\hline 1. Comportamiento directivo & $3.105 \pm 1.037$ & $3.193 \pm 1.104$ & 1.188 & .998 & .318 & .233 \\
2. Implicación club & $2.859 \pm 1.154$ & $3.090 \pm 1.140$ & 8.127 & 6.216 & .013 & $.583^{* *}$ \\
3. Apoyo padres & $3.998 \pm .812$ & $4.133 \pm .804$ & 2.790 & 4.294 & .039 & $.484^{* *}$ \\
4. Presión padres & $2.115 \pm 1.075$ & $2.287 \pm 1.145$ & 4.548 & 3.551 & .060 & $.440^{* *}$ \\
5. Clima tarea comp. & $4.206 \pm .725$ & $4.178 \pm .749$ & .123 & .221 & .638 & -.110 \\
6. Clima ego comp. & $3.334 \pm 1.079$ & $3.333 \pm 1.094$ & .000 & .000 & .991 & -.003 \\
7. Clima tarea entrenador & $4.201 \pm .806$ & $4.326 \pm .649$ & 2.380 & 5.094 & .024 & $.527^{* *}$ \\
8. Clima ego entrenador & $2.260 \pm 1.049$ & $2.218 \pm .980$ & .273 & .276 & .600 & -.123 \\
\hline
\end{tabular}

${ }^{*} p<.05 ;{ }^{* *} p<.01$

\section{Discusión}

El objetivo del presente trabajo era conocer la importancia de los otros significativos, padres, entrenadores y compañeros, sobre la persistencia o abandono de la práctica deportiva en una muestra de jóvenes practicantes de deportes colectivos en edad escolar. En primer lugar, se planteó como hipótesis que un mayor apoyo e implicación de los padres en la práctica deportiva estaría asociado con una mayor continuidad de los jóvenes participantes. Si se observa el análisis de varianza realizado, se aprecia cómo aquellos individuos que continuaron en la práctica presentaban significativamente valores superiores en el apoyo de los padres que los que abandonaron. Asimismo, tras apreciar el análisis discriminante emergen el apoyo y la implicación como fuertes predictoras de la continuidad en la práctica deportiva. En este sentido, es destacable cómo los padres son los que influyen en mayor medida sobre esta continuidad, por encima de entrenadores y compañeros, lo que nos conduce a comprender la importancia que adquiere el núcleo familiar en la adquisición de una práctica deportiva continua en etapas de iniciación.

Estos resultados son consistentes con los hallados por Duda y cols. (2005) y Jowett y Timson-Katchis (2005) quienes encontraron que el feedback positivo, aumento de opor- tunidades y apoyo emocional de los padres está directamente relacionado con la cantidad y calidad de la práctica deportiva de sus hijos/as. En esta línea, Brustad (1996) y Scanlan y Lewthwaite (1986) hallaron una asociación entre el apoyo de los padres en el contexto deportivo, la diversión y una práctica deportiva duradera. Asimismo, se ha demostrado que este apoyo de los padres produce una mayor satisfacción en la práctica de sus hijos/as (Torregrosa y cols., 2007; Wuerth, Lee y Alferman, 2004), lo cual está directamente relacionado con la implicación de los jóvenes en su práctica deportiva. Por tanto, tras los análisis efectuados, se puede afirmar que la primera hipótesis se cumple.

En la línea de la influencia de los padres sobre la práctica deportiva de sus hijos/as, se destaca la aparición de la presión como predictor de la continuidad en el deporte. Este resultado se puede explicar mediante la importancia que adquieren los jugadores con mayores niveles de habilidad, que normalmente son más presionados y debido precisamente a esa alta capacidad no abandonan la práctica. Asimismo, esta orientación de la presión no se muestra de forma explícita, sino que el joven deportista se puede sentir presionado para mejorar, generar hábitos deportivos, no faltar a los entrenamientos... Lo cual no es negativo para la continuidad en la práctica deportiva (González Suárez y Otero Parra, 2005). 
En segundo lugar, se postuló que la continuidad en la práctica deportiva iba a estar asociada positivamente con clima tarea del entrenador y de los compañeros. Si se observa el análisis de varianza, nos indica que existen diferencias significativas entre los que abandonan y persisten en el deporte en función del clima tarea del entrenador. Por el contrario, ninguna de las dos variables que hacen referencia a los compañeros (clima tarea y clima ego), presentan ninguna diferencia con respecto al abandono.

Con respecto al entrenador, Olympiou, Jowett y Duda (2008) demostraron la fuerte asociación entre la importancia que el entrenador da al esfuerzo, colaboración e importancia de cada miembro del equipo y el compromiso deportivo de los jóvenes en edad escolar. Otros trabajos han destacado la importancia del liderazgo del entrenador (Balaguer y cols., 2002; Smith y cols., 2005) como significativo que fomenta comportamientos positivos en los jóvenes en edad escolar. Sin embargo, y contrario a los hallazgos de estudios anteriores (ver Cecchini-Estrada, Méndez y Contreras, 2005; Duda, 2005), en este trabajo no se ha encontrado ninguna diferencia significativa ni predictiva del clima ego del entrenador sobre la persistencia o abandono de la práctica deportiva. Esto nos sugiere la mayor importancia de las otras variables analizadas como la presión, apoyo e implicación de los padres, y el clima tarea del entrenador como herramientas para modular comportamientos en los jóvenes deportistas.

En esta línea, también nos resulta sorprendente que los compańeros de juego, tampoco aparezcan como predictores de la continuidad o el cese de la práctica deportiva. Contrariamente a los estudios expuestos en el marco teórico (Moreno Murcia y cols., 2008; Ntoumanis y cols., 2002), donde se reflejaba la importancia de los compañeros de juego en predecir conductas adaptativas o desadaptativas. Por todo ello, se puede afirmar que la segunda hipótesis no se cumple en su totalidad.
Tras el análisis de los resultados, la principal conclusión que se puede extraer del trabajo es la importancia que adquieren los otros significativos en la adopción de un comportamiento adaptativo como es la persistencia en el deporte. De esta manera, se sugiere que los padres fomenten el apoyo y la implicación al club de sus hijos/as, mientras que los entrenadores y compańeros de juego desarrollen valores como el esfuerzo, progresión, diversión en las tareas de aprendizaje... como medio de generar un clima de aprendizaje positivo y que conduzca a no abandonar la práctica deportiva.

En cuanto a las limitaciones del estudio, se destaca que se han analizado a todos los deportistas y los otros significativos de forma conjunta. De esta manera, una valoración por deporte mediante el análisis multinivel, nos daría información más detallada de las relaciones entre ellos. Asimismo, consideramos que la realización de análisis diádico para valorar por pares, nos ayudaría mucho más en la compresión de la influencia que ejerce el entorno sobre el joven deportista. Para finalizar este apartado, se indica que la utilización del cuestionario para valorar el clima motivacional percibido en los compañeros de juego no está validado en España, por lo que podría presentar algún sesgo, debido principalmente a aspectos culturales.

Igualmente, se considera interesante el establecimiento de prospectivas de futuro para ayudarnos a continuar y aumentar el conocimiento acerca de las causas y motivos que conducen a la persistencia y el abandono deportivo. De esta manera, el aumentar el período de duración de valoración de la variable abandono, aumentaría la fiabilidad del estudio. El incremento de la muestra y los deportes analizados, también nos proporcionaría mucha más información al respecto. Por otro lado, la realización de diseńos experimentales, mediante la aplicación de estrategias motivacionales y de aumento del compromiso, nos ayudaría a entender mejor las razones de la persistencia y el cese de los jóvenes en edad escolar.

\section{Referencias bibliográficas}

Williams, J. (1991). Características psicológicas del alto rendimiento. En Williams, J. (Ed.) Psicología aplicada al deporte. Madrid: Biblioteca Nueva.

Ames, C. (1992). Achievement goals, motivational climate, and motivational processes. En G. C. Roberts (Ed.), Motivation in sport and exercise (pp. 161-176). Champaign, IL: Human Kinetics.

Balaguer, I., Duda, J. L., Atienza, F. L., y Mayo, C. (2002). Situational and dispositional goals as predictors of perceptions of individual and team improvement, satisfaction and coach ratings among elite female handball teams. Psychology of Sport and Exercise, 3, 293-308.

Balaguer, I., Duda, J. L., y Crespo, M. (1999). Motivacional climate and goal orientations as predictors of perceptions of improvement, satisfaction and coach ratings among tennis players. Scandinavian Journal of Medicine and Science in Sports, 9, 381-388.

Bartholomew, K., Ntoumanis, N., y Thogersen-Ntoumani, C. (2010). The controlling interpersonal style in a coaching context: Development and initial validation of a psychometric scale. Journal of Sport and Exercise
Psychology, Journal of Sport and Exercise Psychology, 32, 193-216.

Boixadós, M., Cruz, J., Torregrosa, M., y Valiente, L. (2004). Relationship among motivational climate, satisfaction, perceived ability and fair play attitudes in young soccer players. Journal of Applied Sport Psychology, 16, 301-317.

Brustad, R. J. (1996). Parental and peer influence on children's psychological development through sport. En F. L. Smoll y R. E. Smith (Eds.), Children and youth in Sport - A Biopsychosocial Perspective (pp. 112-155). Washington: Brown \& Benchmark.

Cecchini Estrada, J. A., Méndez, A., y Contreras Jordán, O. (2005). Motivos de abandono de la práctica del deporte juvenil. Universidad de Castilla La Mancha: Cuenca.

Cervelló, E. M., Escarti, A., y Guzmán, J. F. (2007). Youth sport dropout from the achievement goal theory. Psicothema, 19, 65-71.

Conroy, D. E., y Coatsworth, J. D. (2006). Coach training as a strategy for promoting youth social development. The Sport Psychologist, 20, 128144. 
Duda, J. L. (2005). Motivation in Sport: The Relevance of Competence and Achievement Goals. In A. J. Elliot y C. S. Dweck (Eds.), Handbook of competence and motivation (pp. 273-308). New York: Guilford Publications.

Duda, J. L., Ntoumanis, N., Mahoney, J. L., Larson, R. W., y Eccles, J. S. (2005). After-school sport for children: Implications of a task-involving motivational climate. Mahwah, NJ, US: Lawrence Erlbaum Associates, Publishers.

Ebel, R., y Frisbie, D. (1986). Essentials of Education Measurement. Englewood Cliffs, NJ: Prentice Hall.

Ferrer-Caja, E., y Weiss, M. R. (2002). Cross-validation of a model of intrinsic motivation in physical education with students enrolled in elective courses. Journal of Experimental Education, 71, 41-65.

Fraser-Thomas, J., y Côté, J. (2009). Understanding adolescents' positive and negative developmental experiences in sport. The Sport Psychologist, 23, 3-23.

Gallimore, R., y Tharp, R. (2004). What a coach can teach a teacher, 19752004: Reflections and reanalysis of John Wooden's teaching practices. The Sport Psychologist, 18, 119-137.

García Calvo, T., Leo, F. M., Martín, E., y Sánchez Miguel, P. A. (2008). El compromiso deportivo y su relación con factores disposicionales y situacionales de la motivación. Revista Internacional de Ciencias del Deporte, 4(12), 45-58.

Glass, G., y Stanley, J. (1986). Métodos estadísticos aplicados a las ciencias sociales.México: Prentice Hall Hispanoamericana.

González Suárez, A. M., y Otero Parra, M. (2005). Actitudes de los padres ante la promoción de la actividad física y deportiva de las chicas en edad escolar. Cuadernos de Psicología del Deporte, 5 (1 y 2).

Jowett, S., y Timson-Katchis, M. (2005). Social Networks in Sport: Parental Influence on the Coach-Athlete Relationship. Sport Psychologist, 19(3), 267-287.

Lee, M. J., y McLean, S. (1997). Sources of parental pressure among age group swimmers. European Journal of Physical Education, 2, 167-177.

Mageau, G. A., y Vallerand, R. J. (2003). The coach-athlete relationship: A motivational model. Journal of Sports Sciences, 21, 881-954.

Moreno Murcia, J. A., López de San Román, M., Martínez, C., Alonso, N., y González-Cutre, D. (2008). Peers influence on exercise enjoyment: A self-determination theory approach. Journal of Sports Science and Medicine, 7, 23-31.

Newton, M. L., Duda J. L., y Yin, Z. (2000). Examination of the psychometric properties of the Perceived Motivational Climate in Sport Questionnaire-2 in a sample of female athletes. Journal of Sports Sciences, $18,275-290$.

Nicholls, J. G. (1989). The competitive ethos and democratic education. Cambridge, MA, US: Harvard University Press.

Nicholls, J. G. (1992). The general and the specific in the development and expression of achievement motivation. En G.C. Roberts (Ed.), Motivation in sport and exercise (pp.57-91). Champaign, IL: Human Kinetics.

Ntoumanis, N., Vazou, S., y Duda, J. L. (2007). Peer-Created Motivational Climate. [Book; Edited Book] Jowette, Sophia (Ed); Lavallee, David (Ed). Social Psychology in Sport. (pp. 145-156). Champaign, IL, US: Human Kinetics.

Ntoumanis, N., y Vazou, S. (2005). Peer motivational climate in youth sport: measurement development and validation. Journal of Sport and Exercise Psychology, 27, 432-455.

Olympiou, A., Jowett, S., y Duda, J. L. (2008). The psychological interface of the coach-created motivational climate and the coach-athlete relationship. The Sport Psychologist, 22, 423-438.

Ommundsen, Y., Roberts, G. C., Lemyre, P. N., y Miller, B. W. (2005).
Peer relationships in adolescent competitive soccer: Associations to perceived motivational climate, Achievement goals and perfectionism. Journal of Sports Sciences, 23, 977-989.

Ommundsen, Y., y Roberts, G. C. (1999). Effect of motivational climate profiles on motivational indices in team sport. Scandinavian Journal of Medicine and Science in Sports, 9, 389-397.

Papaioannou, A. G., Ampatzoglou, G., Kalogiannis, P., y Sagovits, A. (2008). Social agents, achievement goals, satisfaction and academic achievement in youth sport. Psychology of Sport and Exercise, 9, 122-141.

Sarrazin, P., Vallerand, R., Guillet, E., Pelletier, L., y Cury, F. (2002). Motivation and dropout in female handballers: A 21-month prospective study. European Journal of Social Psychology, 32, 395-418.

Scanlan, T. K., y Lewthwaite, R. (1984). Social psychological aspects of competition for male youth sport participants: IV. Predictors of performance outcomes. Journal of Sport Psychology, 6, 422-429.

Smith, A. L., Balaguer, I., y Duda, J. L. (2006). Goal orientation profile differences on perceived motivational climate, perceived peer relationships, and motivation-related responses of youth athletes. Journal of Sports Sciences, 24(12), 1315-1327.

Smith, S. L., Fry, M. D., Ethington, C. A., y Li, Y. (2005). The effect of female athletes' perceptions of their coaches' behaviours on their perceptions of motivational climate. Journal of Applied Sport Psychology, 17, 170-177.

Sousa, C., Arevalo, A., Ramis, S., Torregrosa, M., Viches, D., y Cruz, J. (2006). Design and implementation of coach training program in youth soccer. En Abstracts of 26th International Congress of Applied Psychology (p. 984) [CD], Greece: International Association of Applied Psychology.

Tammelin, T., Näyhä, S., Hillas, A. P., y Järvelin, M. J. (2003). Adolescent participation in sports and adult physical activity. American Journal of Preventive Medicine, 24, 22 - 28.

Telama, R., Yang, X., Viikari, J., Välimäki, I., Wanne, O., y Raitakari, O. (2005). Physical activity from childhood to adulthood: A 21-year tracking study. American Journal of Preventive Medicine, 28(3), 267 - 273.

Torregrosa, M., Cruz, J., Sousa, C., Viladrich, C., Villamarín , F., GarcíaMas, A., y Palou, P. (2007). La influencia de padres y madres en el compromiso deportivo de futbolistas jóvenes. Revista Latinoamericana de Psicología, 39(2), 227-237.

Torregrosa, M., Figueroa, J., Garcia-Mas, A., Sousa, C., Vilches, D., Villamarín, F., y Cruz, J. (2005, Mayo). La relación entre las familias (padres y madres) y el compromiso deportivo de futbolistas cadetes. Comunicación presentada al VII Congreso Sudamericano de Psicología del Deporte la Actividad Física y la Recreación, Montevideo, Uruguay.

Vanreusel, B., Renson, R., Beunen, G., Claessens, A. L., Lefevre, J., Lysens, L., y Vanden Eynde, B. (1997). A longitudinal study of youth sport participation and adherence to sport in Adulthood. International Review for the Sociology of Sport, 32 (4), 373 - 387.

Weiss, M. R., y Duncan, S. C. (1992). The relationship between physical competence and peer acceptance in the context of children's sports participation. Journal of Sport and Exercise Psychology, 14, 177-191.

Weiss, M. R., y Fretwell, S. D. (2005). The parent-coach/child-athlete relationship in youth sport: Cordial, contentious, or conundrum? Research Quarterly for Exercise and Sport, 76, 286-305.

Weiss, M. R., y Smith, A. L. (2002). Friendship quality in youth sport: Relationship to age, gender, and motivation variables. Journal of Sport y Exercise Psychology, 24, 420-437.

Wuerth, S., Lee, M., y Alfermann, D. (2004). Parental Involvement and athletes career in youth Sport. Psychology of Sport and Exercise, 5, 21-33. 